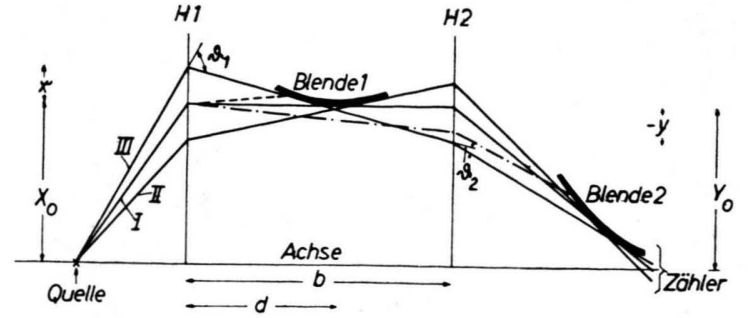

Abb. 1. Schematischer Strahlengang. H 1 (H 2) Hauptebene von Linse 1 (2). I, II und III bezeichnen drei Bahnen verschiedenen Achsenwinkels; ausgezogen: $p=p_{0}$; gestrichelt : $p>p_{0}$; strichpunktiert: $p<p_{0}$. Die eingezeichneten Strekken $x$ und $y$ beziehen sich auf Bahn III.

$$
\frac{\beta_{2}}{1-b / d}+\gamma_{2}(1-b / d)^{2}>0 ;
$$

$d$ ist der Abstand des Berührungspunktes von Elektronenbahn $\left(p=p_{0}\right)$ und Blende 1 von der Hauptebene $\mathrm{H} 1$.

Für dünne Linsen gelten die folgenden Näherungsformeln ${ }^{3}$ :

$$
f=\frac{2}{\pi} \frac{a}{k^{2}}, \quad C_{\ddot{0}}=\frac{2}{\pi^{3}} \frac{a}{k^{6}} ;
$$

3 W. Glaser, Z. Phys. 117, 285 [1941]. $f=$ Brennweite, $k=H_{0} a /(2 H \varrho)$ mit $H_{0}=$ Feldstärke im Linsenzentrum, $2 \boldsymbol{a}=$ Halbwertbreite der Feldverteilung auf der Achse und $H \varrho=$ Steifigkeit der Elektronen, $C_{\ddot{o}}=$ Öffnungsfehlerkonstante.

Damit gehen die Gln. (I), wenn man etwa $a_{1}=a_{2}$ und $1-b / d=-2$ macht, über in

$$
\begin{aligned}
& -\frac{1}{2} k_{1}^{2}+4 k_{2}^{2}>0 \text {, } \\
& \frac{1}{2} k_{1}{ }^{2}-k_{2}{ }^{2}>0 \text {, } \\
& k_{2}^{2}\left(1+\frac{3}{4} \frac{X_{0}{ }^{2}}{a_{1}{ }^{2}}\right)-\frac{3}{8} \frac{X_{0}{ }^{2}}{a_{1}{ }^{2}} k_{1}{ }^{2}>0,
\end{aligned}
$$

und man überzeugt sich, daß diese Forderungen (II) erfüllt sind etwa mit $k_{1}=2 k_{2}$ und $X_{0} \ll a_{1}$; der Index 1 (2) bezieht sich dabei auf die Linse 1 (2).

Der Vollständigkeit halber sei noch erwähnt, daß man die Reihenfolge der Linsen 1 und 2 vertauschen kann; diese Variante hat aber den Nachteil kleinerer Lichtstärke.

In praxi wird man als erste Linse eine dicke Linse nehmen, da die sphärische Aberration dicker Linsen geringer ist. Die Verhältnisse werden dann unübersichtlicher, und man ist vollständig auf numerische Bahnberechnungen oder photographische Bahnbestimmungen angewiesen.

Der Aufbau des Geräts wird sich etwas schwierig gestalten, so daß die aufgewandte Mühe sich nur dort lohnen wird, wo es bei hoher Auflösung extrem auf die Lichtstärke ankommt, etwa bei einem Paarspektrometer.

\section{Zur Messung von Dampfstrahldichten mittels Ionisationsmanometer beim Aufdampfen dünner Schichten}

Von O. HaAse

Institut für Angewandte Physik der Universität Hamburg (Z. Naturforschg. 12 a, 941-942 [1957]; eingegangen am 18. September 1957)

Bei der Herstellung dünner Schichten liegt die Aufgabe vor,

1. vorgegebene Mengen von Substanzen auf eine Unterlage aufzubringen (z. B. um die Abhängigkeit der Struktur von der Schichtdicke zu studieren) und

2. die Aufdampfgeschwindigkeit definiert zu variieren.

Die erste Frage läßt sich im Prinzip dadurch lösen, daß die Schichtdicke z. B. mit einem Interferenzmikroskop gemessen wird. Will man jedoch diese Werte bereits während des Aufdampfens, z. B. in der Elektroneninterferenźapparatur messen, so stößt man auf Schwierigkeiten. Zur Kontrolle der Aufdampfgeschwindigkeit existiert kein geeignetes Meßverfahren, das für die allgemein verwendeten Substanzen brauchbar ist. Ein einfaches und quantitatives Verfahren bietet sich durch folgende Anordnung.

Als Meßsystem wurde eine kommerzielle Ionisationsmanometer-Röhre verwendet, deren System neben der zu bedampfenden Unterlage aufgestellt ist und über eine rotierende Blende periodisch dem Dampfstrahl ausgesetzt wird (Abb. 1). Die in das Ionisationsmanometer eindringenden Dampfmoleküle werden von dem Elektronenstrom ionisiert. Wenn die Aufdampfsubstanz auf den Gefäßwänden haftet, wie es bei den meist benutzten Schichtsubstanzen der Fall ist, wird die Dichte im Ionisierungsraum des Manometers periodisch schwan-
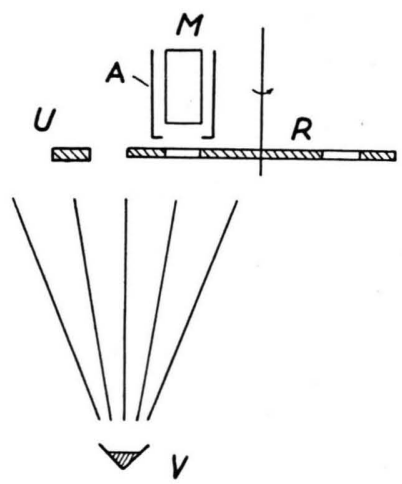

Abb. 1. Anordnung zur Messung der Aufdampfgeschwindigkeit mit einem Ionisationsmanometer. U $\mathrm{Zu}$ bedampfende Unterlage, M Ionisationsmanometer-Röhre, A Abschirmung, $\mathrm{R}$ Rotierende Blende, $6 \mathrm{~Hz}$ Blendenfrequenz, V Aufdampfofen. 


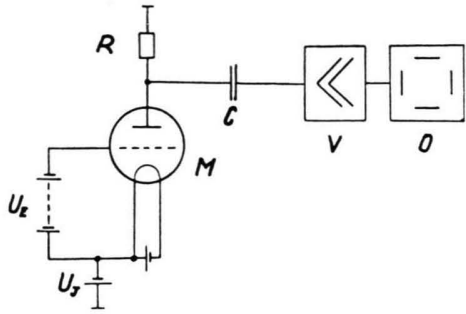

Abb. 2. Schaltung des Ionisationsmanometers zur Messung von Aufdampfgeschwindigkeiten. M IonisationsmanometerRöhre, $\mathrm{U}_{\mathrm{E}}$ Beschleunigungsspannung für die Elektronen $100 \mathrm{~V}$, UJ Auffängerspannung $30 \mathrm{~V}$, R Arbeitswiderstand des Auffängers $1 \mathrm{M} \Omega$, C Koppelkondensator $0,1 \mu \mathrm{F}$, V Niederfrequenzverstärker, 2-stufig, O Oszillograph mit Verstärker $50 \mathrm{mV} / \mathrm{cm}$.

ken. Die dadurch hervorgerufene Wechselstromkomponente des Ionenstromes, die um Größenordnungen kleiner als die Gleichstromkomponente des Restvakuums (in den vorliegenden Versuchen $5 \cdot 10^{-5}$ Torr) sein darf, wird am Arbeitswiderstand des Ionenfängers kapazitiv abgenommen und nach Verstärkung mit einem Oszillographen gemessen. Abb. 2 zeigt die elektrische Schaltung. An den Aufdampfofen wurde eine positive Spannung von 150 Volt gelegt, um zu verhindern, daß vom Aufdampfofen emittierte Elektronen auf den Ionenfänger gelangen.

Als Aufdampfofen wurde ein Tantalschiff verwendet, das mit einer Silbermenge für eine Schichtdicke von 100 Å beschickt war. Der Aufdampfofen wurde so einreguliert, daß die Wechselspannungskomponente des Ionenstromes am Widerstand $R$ (s. Abb. 2) $100 \mu \mathrm{V}$ betrug; siehe Abb. 3, große Amplitude. Mit diesem Silbervorrat lief der Ofen bei konstanter Aufdampfgeschwindigkeit, erkenntlich an der Konstanz der Amplitude, $100 \mathrm{sec}$, was einer Aufdampfgeschwindigkeit

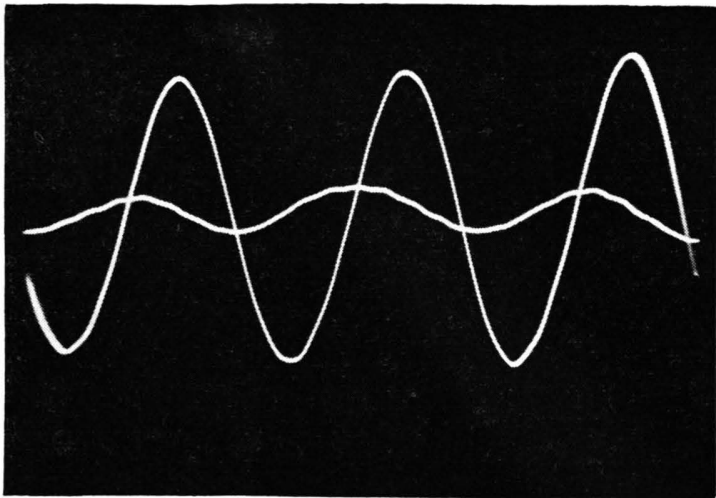

Abb. 3. Oszillogramm der Wechselstromkomponente des Ionenstromes einer Ionisationsmanometer-Röhre, die von einem modulierten $(6 \mathrm{~Hz})$ Dampfstrahl getroffen wird. Große Amplitude: Silberdampfstrahl entsprechend einer Aufdampfgeschwindigkeit von $1 \AA /$ sec. Kleine Amplitude: Nullverlauf, nachdem alles $\mathrm{Ag}$ verdampft ist und der leere Verdampfungsofen mit gleicher Stromstärke weiter betrieben wird.

von $1 \AA \AA$ Asec entspricht. Sobald alles Silber verdampft war, ging auch der Ausschlag auf dem Oszillographen auf den Ruhewert zurück (Abb. 3, kleine Amplitude, $15 \mu \mathrm{V}$. Der Amplitude von $10 \mu \mathrm{V}$ entspricht ein Druck von etwa $10^{-9}$ Torr). Um die obige Abschätzung zu prüfen und die Apparatur zu eichen, wurde die Dicke des Silberniederschlages in einem Vielstrahlinterferenzmikroskop (C. E. Joh a n s s o n, Schweden) nachgemessen. Mit der Anordnung können auch Schichten so geringer Dicke definiert hergestellt werden, die mit dem Dickenmeßverfahren selbst nicht mehr gemessen werden können. Über die Untersuchung der so hergestellten Schichten wird später zusammenfassend berichtet.

\section{Über den Bandabstand von Galliumnitrid und Aluminiumnitrid}

Von E. KaUer und A. Rabenau

Allgemeine Deutsche Philips Industrie G.m.b.H., Zentrallaboratorium Aachen

(Z. Naturforschg. 12 a, 942-943 [1957]; eingegangen am 7. Oktober 1957)

Die unzulängliche Kenntnis der Halbleitereigenschaften einiger III - V-Verbindungen beruht vor allem auf den technologischen Schwierigkeiten, die der Herstellung von Einkristallen entgegenstehen. Eine wesentliche Größe, nämlich der Bandabstand $\Delta E$ und dessen Temperaturabhängigkeit läßt sich jedoch mittels Remissionsmessungen auch an polykristallinen Proben bestimmen. Das Meßverfahren beruht auf einem Vergleich der spektralen Intensitäten $I$ und $I_{0}$, die von der zu untersuchenden Probe und einer MgO-Schicht als Standard reflektiert werden. Trägt man als Maß für die Absorp- tion $\log \left(I_{0} / I\right)$ gegen die Photonenenergie in $\mathrm{eV}$ auf, so erhält man Kurven, die im Bereich der Absorptionskante angenähert geradlinig verlaufen. $\mathrm{Da}$ das von Macfarlane und Roberts ${ }^{1}$ angegebene Verfahren zur genauen Festlegung der Kante auf den vorliegenden Fall nicht anwendbar ist, erscheint es sinnvoll, als Einsatzpunkt der Absorption den Schnittpunkt des extrapolierten Steilanstiegs mit dem Pegel der Restabsorption zu definieren.

GaN und AlN haben im Gegensatz zu den in Zinkblende-Struktur kristallisierenden III - V-Verbindungen Wurtzit-Struktur, worin der höhere Anteil an Ionenbindung zum Ausdruck kommt. Sie werden hergestellt durch Umsetzen der Metalle mit sorgfältig gereinigtem Ammoniak bei $1100{ }^{\triangleleft} \mathrm{C}\left(\mathrm{GaN}^{2}\right)$ bzw. 1400 bis $1500{ }^{\circ} \mathrm{C}(\mathrm{AlN})$.

1 G.G. Macfarlane u. V.Roberts, Phys. Rev. 97, 1714 [1955].

2 H. HahN u. R. Juza, Z. anorg. allg. Chem. 244, 111 [1940]. 